\title{
Spacetime as an Emergent Phenomenon: A Possible Way to Explain Entanglement and the Tunnel Effect
}

\author{
P. Castro' ${ }^{1}$, M. Gatta ${ }^{1,2}$, J. R. Croca ${ }^{1,3}$, R. Moreira ${ }^{1}$ \\ ${ }^{1}$ Center for Philosophy of Sciences of the University of Lisbon (CFCUL), Lisbon, Portugal \\ ${ }^{2}$ CINAV and Escola Naval (Portuguese Naval Academy), Lisbon, Portugal \\ ${ }^{3}$ Department of Physics, Faculty of Sciences, University of Lisbon, Lisbon, Portugal \\ Email: jpcastro@fc.ul.pt
}

How to cite this paper: Castro, P., Gatta, M., Croca, J.R. and Moreira, R. (2018) Spacetime as an Emergent Phenomenon: A Possible Way to Explain Entanglement and the Tunnel Effect. Journal of Applied Mathematics and Physics, 6, 2107-2118. https://doi.org/10.4236/jamp.2018.610177

Received: May 21, 2018

Accepted: October 23, 2018

Published: October 26, 2018

Copyright $\odot 2018$ by authors and Scientific Research Publishing Inc. This work is licensed under the Creative Commons Attribution International License (CC BY 4.0).

http://creativecommons.org/licenses/by/4.0/

\begin{abstract}
Entanglement and the tunnel effect phenomena have been repeatedly observed and are generically accepted under orthodox quantum mechanics formalism. However, they remain rather inexplicable in the context of spacetime usual conceptualization. In the present work, we suggest an alternative quantum mechanics formalism, refining the pilot-wave theory initially proposed by de Broglie. We suggest that spacetime is an emergent phenomenon from a prior subquantum medium and that entanglement and the tunnel effect can be explained in terms of a nonlinear relation between space and time that is imposed by subquantum waves.
\end{abstract}

\section{Keywords}

Spacetime, Emergent Phenomena, Entanglement, Tunnel Effect, Subquantum Medium, Nonlinear Relation, Subquantum Wave, Superluminal Velocities

\section{Introduction: From De Broglie Pilot-Wave Theory to a Pilot-Wavelet Model}

Over the last century, quantum mechanics brought about very serious concerns about the inner nature of space and time. Several experimental situations have strongly suggested that, somehow, a certain degree of ubiquity characterizes atomic entities. The Copenhagen School, championed by Niels Bohr, solved the problem postulating the meta-physical existence of probability waves, the so-called psi waves. These would be space and time infinitely spread fields, immediately destroyed once an observation occurs, in the so-called wave collapse 
or state vector reduction. The atomic entity then appears from "nowhere", since it had been only potentially waiting to come into existence before measurement has been performed. This somehow exotic and metaphysical way of describing Reality soon became the only accepted tool to deal with what seemed to be $\mathrm{Na}$ ture's ubiquity at the atomic level. Indetermination of the quantum entities trajectories, far from the pure idealistic exactness of Newton's and Einstein's kinematics, as well as some observable bizarre behaviors, like entanglement and quantum tunneling, is the key ingredients to support the beliefs of contemporary orthodoxy thought on the subject.

Such seemingly quantum phenomena have thus been used to dismiss criticism from holding an alternative realistic view about Nature. The first critic was an early one. Already in 1927, at the celebrated Solvay conference on Physics, Louis de Broglie proposed that atomic entities would exist in space and time at all times, being guided or piloted by what he called subquantum waves [1]. Unfortunately, de Broglie opponents, most specially Bohr, adopted Heisenberg's view and applied Fourier's analysis to package the train waves into a localized corpuscle. This, of course, included pure harmonic waves spreading infinitely trough space and time. Niels Bohr was very much aware of the difficulty of allowing real physical perturbations to extend infinitely throughout the Universe and, quite wisely, dismissed them as such, adopting his psi wave platonic discourse. However, still a lot of perplexing questions remained to be answered. Where exactly do such platonic probability-waves stand and how do they interact with physical objects like mirrors, slits and barriers, without collapsing? Who did collapse the waves before humans or any other creatures were standing? How does this happen? If collapse is brought about by environment noise, does this mean that quantum phenomena are a rare one in the Universe and so, largely irrelevant as such? Finally, and mostly important to the topic at hand, why does it seem that quantum phenomena allow for the occurrence of superluminal phenomena, the proverbial Einstein's claim against spooky actions at distance?

So, we can highlight some traditional questions in the foundations of quantum mechanics, as usually interpreted, namely in the Copenhagen tradition.

First, there is the problem that this orthodox quantum mechanics lacks intelligibility, as it accepts infinite metaphysical waves to perform physical actions.

Second, as we try to derive spacetime, adventuring ourselves into more and more abstract pictures severed from observable physical reality, we risk ending up with mere formal mathematical games.

A philosophical turn into some sort of Objective Reality seems therefore desirable, in order to return to a more tangible world. This was, in a sense, what de Broglie had in mind.

The original pilot-wave model de Broglie envisaged was refined many years later [2] with the very substantial modification of replacing the usual sine and cosine waves used in Fourier common analysis by Morlet wavelets [3] [4] [5] [6]. A wavelet is then employed as a possible and convenient formal representation of a finite subquantum perturbation, reducing to the usual harmonic plane wave 
once the associated $\sigma$ parameter, representing the wavelet spatial extension, becomes very large. That is, whenever $\sigma \rightarrow \infty$, one obtains:

$$
\theta=A \mathrm{e}^{-\frac{(x-v t)^{2}}{2 \sigma_{0 x}^{2}}+i(k x-\omega t)} \underset{\sigma_{0 x} \rightarrow \infty}{\longrightarrow} \theta=A \mathrm{e}^{i(k x-\omega t)}
$$

The expression on the right is, in fact, the usual mathematical expression for the harmonic plane wave, spreading infinitely over space and time, that is, the abstract case resulting from the more general and realistic wavelet expression, on the left side of (1).

Another very important feature is that the wavelet expression is formally a solution of a nonlinear differential equation, called the Master equation, see ref. [2], reading:

$$
-\frac{\hbar^{2}}{2 \mu} \nabla^{2} \theta+\frac{\hbar^{2}}{2 \mu} \frac{\nabla^{2}\left(\theta \theta^{*}\right)^{1 / 2}}{\left(\theta \theta^{*}\right)^{1 / 2}} \theta+V \theta=i \hbar \frac{\partial \theta}{\partial t}
$$

Here, $\hbar$ is Planck's constant, $\mu$ represents the particle mass, $V$ its potential energy and $\theta$ the physically existent quantum wavefunction. It should be noted that whenever the second term in the left part of the equality, the so called nonlinear term, is null or constant, one obtains the linear Schrödinger differential equation.

It is quite remarkable that the wavelet is a non-dispersive solution of the Master equation and has determined temporal frequency $\omega$ and spatial frequency $k$ at all times, even though it does not spread throughout all of space and time. This fact has major consequences to what is to be thought about the nature of physical entities at quantum scales. In fact, a set of generalized uncertainty relations can be obtained using wavelet composition [7] [8] [9]. As a matter of fact, following a procedure very much analogous to the one used by Niels Bohr in his famous 1927 Como lecture paper [10], and which was based on standard Fourier analysis. The generalized uncertainty relations [11] [12] read:

$$
\begin{aligned}
& \Delta p_{x}=\frac{\hbar}{\Delta x} \sqrt{1-\Delta x^{2} / \sigma_{0 x}^{2}} \\
& \Delta E=\frac{\hbar}{\Delta t} \sqrt{1-\Delta t^{2} / \sigma_{0 t}^{2}}
\end{aligned}
$$

where $\Delta p_{x}$ is the maximum interval for all the possible linear momentum values of the system being observed, traditionally termed the linear momentum error or the linear momentum uncertainty, and where $\Delta x$ is the maximum interval for all the possible space coordination values of the system being observed, traditionally termed the position error or the position uncertainty. $\Delta E$ is the energy maximum interval and $\Delta t$ is the maximum time interval, similarly to the above. And, finally, where we have provisionally considered $\sigma_{0 x}=v \sigma_{0 t}$ in the second relation, $\sigma_{0 x}$ represents the spatial extension of the mother wavelet used in the wavelet packaging, $v$ being the composite wavelet average velocity.

Once more, when $\sigma \rightarrow \infty$ one obtains the usual Heisenberg relations. The 
wavelet description is therefore formally more general than traditional harmonic plane waves of orthodox quantum mechanics. Furthermore, it provides a very natural picture for real limited subquantum undulatory perturbations, introducing in this way a new element of realism in the quantum mechanics narrative. Moreover, it is a pilot-wave model because it assumes that each corpuscular system has its own overall associated subquantum wave, a finite perturbation propagating through space and time. The motion of the inter-relational complex corpuscle will be such, that, on average, it will tend to move to the regions where the intensity of the guiding wavelet is greater. This statement, named the Principle of Eurhythmy, constitutes an equivalent formulation to the "Guidage" Guiding Principle, introduced by de Broglie [13]. Finally, as an element of realism, namely the subquantum waves, has been restored into physical discourse, thus providing a possible unifying description of reality, one can extend the use of the wavelet formalism to all scales. In that way, it is expected that gravitational systems will also exhibit undulatory properties, which is, in fact, the case with the Titius-Bode regularity, observable in our Solar System [14] and eventually in many other planetary systems across the universe. We call our model Eurhythmic Physics, since any Guidage acting upon systems, as in the extreme principle cases (e.g. Fermat) seem to suggest that entities follow a preferable path over other possible paths. And so, these entities are, in fact, moving along the best possible path in order to endure. This is the principle of Eurhythmy, from the Greek euritmia, which is the composition of the root eu plus rhythmy: eu standing for the right, the good, the adequate and rhythmy, for the way, the path, the harmonic motion [15] [16] [17].

\section{The Spacetime Language Problem and the Minimum Description Method}

The pilot-wavelet realistic model seems to provide an adequate solution to at least some of the daunting questions orthodox quantum mechanics left unsolved. Since particles and waves exist simultaneously at all times, there will be no collapse by means of the mysterious conscience powers attributable to human observers, or by means of a strange decoherence from environment noise, acting over a metaphysical probability psi wave. However, one last difficult remains the superluminal character of quantum phenomena. This is the problem that we will address here.

We shall follow de Broglie's original insights that a subquantum medium should be thought to be the most fundamental physical element of reality. A kind of sub-atomic realm from which the first and most elementary structures form, arising from what otherwise can only be described as an undefined medium. Since it is plausible to accept that quantum entanglement and quantum tunneling suggest that the concepts of space and time are derivable from a more fundamental reality, it is natural to consider the subquantum medium as such primordial source. However, as it soon becomes obvious, we are again dealing with the same type of problem we acknowledged when we referred to the sym- 
bolic remoteness of Hilbert space from observable reality. The real common difficulty is that human natural language and thoughts are highly embedded in our spatial and temporal experience. Thus, even if we are able to logically, or perhaps, in some cases, intuitively acknowledge a prior reality to space and time, we are like fishes in an aquarium. We are able to see the outer world through the glass walls, but incapable of describing it in terms of the usual inner world experiences available to our senses. Human language and rational thought are operationally grounded in space and time experience, and so, it is extremely hard, if not impossible, to describe a prior reality, without severing it from the very elements of reality, space and time, one wishes to derive. This is, in sum, the spacetime language problem.

A possible solution to this problem is simply to abdicate from describing the inner works of an a priori reality to spacetime and, indeed, to adopt a kind of minimum possible description, focusing our thought on the boundary between the subquantum medium and the emergent spacetime. This implies still using the same spacetime grounded mathematical formalisms, but giving them new meanings and, in particular, allowing for the applied mathematical structures to represent behaviors on what can be called border causal regions. That is, regions where the reality prior to space time and the spacetime emergent reality intersect, showing properties from both reigns. What was herein stated sufficiently define, for now, what can be called the minimum description method. Its application will become clearer as we progress throughout this work. Let us thus begin by stating the following:

1) A subquantum wave is defined as a boundary physical structure, emerging from the subquantum medium, with a minimum degree of order and giving meaning to a spacetime environment, that is, a fundamental reality that can be perceived in terms of observable extensions and perceivable durations.

2) The subquantum wave is a structure that encodes both spacetime properties and subquantum medium properties. The wave's space and time properties are represented respectively, by the wavelength and the frequency. The wave subquantum properties will be called domain and interconnectivity, to be defined as follows.

3) Subquantum properties.

a) There are two subquantum properties: domain and interconnectivity.

i) Domain is defined as the degree of diversity that may be attributed to a set of systems, interacting under a subquantum wave's influence.

ii) Interconnectivity is defined as the degree with which a system interacts and modifies another system, under a subquantum wave's influence.

b) Domain and interconnectivity will be postulated to be subquantum properties, since for certain situations, depending on their numerical values, the usual concept of velocity of a mediating causal agent will not apply.

c) The subquantum property named "domain" will be represented by the subquantum wave maximum spatial extension $\sigma_{0 x}$, defining the longest measurable distance between two interacting systems, under the wave's spatial in- 
fluence. The subquantum property named "interconnectivity" will be represented by the subquantum wave maximum temporal extension $\sigma_{0 t}$, defining the longest measurable time interval of any interaction occurring under the wave's spatial influence.

4) A spacetime environment or spacetime region will be defined as the set of all possible spacetime coordinating values characterizing any interaction under the wave's influence. Spacetime will be characterized by the relation between a given generic space interval and a given generic time interval for a generic interaction under the wave's influence. This will be called a spacetime relation.

5) It will be assumed that the spacetime relation will be the core spacetime property, that is, a characteristic representation of how interactions occur in space and time under the wave's domain of influence. It will also be assumed that the spacetime relation is an emergent property of the subquantum medium, depending on the domain and on the interconnectivity. It will be in this sense that spacetime will be understood as an emergent phenomenon from the subquantum medium.

6) As such, physical reality will be in fact nonlocal at the most fundamental level, the apparent spatial and temporal localities being a common-but not by any means necessary and universal-consequence of the averaging out of the spatial-temporal relations between phenomena allowed by the emergent subquantum waves. In this regard, space and time become relational in a manner somewhat akin to the Leibnizian proposal: we would say that space denotes, in terms of actuality, an order of subquantum waves which exist at the same time, considered as existing together, whereas time would denote an order of succession of subquantum waves overlapping in the space they generated themselves.

\section{Describing Spacetime as an Emergent Phenomenon. The Generic Spacetime Relation Formulation}

We will now use the conceptual tools presented in the last section to build a minimum description capturing spacetime as an emergent phenomenon from the subquantum medium. For that purpose, and following what was posited in 4, we will formulate a spacetime relation from the generalized uncertainty relations (3) and (4). We will then use such generic spacetime relation to derive some conclusions about the entanglement and tunneling phenomena, giving meaning to the statement in 3.2 that, for certain situations, the concept of velocity of a mediating signal will not apply.

With Bohr [10] such packaging involved an infinite number of infinitely spread harmonic waves. This is of course, a direct consequence of Fourier analysis. Since these (psi) waves were assumed to be of a purely meta-physical nature, their infinite character offered no problem. On the other hand, the same metaphysical nature made nonsensical any cinematic description of the particles on a spacetime stage. In the present context, however, an identical packaging procedure will now be performed having on mind real limited subquantum perturbations, represented by Morlet wavelets and with variable extension around a given 
position [11] [12]. These perturbations have physical reality and furthermore exist simultaneously with the material corpuscles, since the former are pilot-waves guiding the movements of the particles. Such understanding of reality makes it then possible to use the generalized uncertainty relations as a tool to analyze cinematic properties of objects, under the overall packaged wave influence. Therefore, the spatial interval $\Delta x$ and the time interval $\Delta t$ can be used as generic space and time parameters that, in most situations (although, as we will see, not all), are linearly related by a velocity, as with $\Delta x \approx v \Delta t$.

The derivation of what will be called a generalized spacetime relation follows. We start by considering the energy of a generic causal agent, propagating during a time $\Delta t$, from a system to another, along a distance $\Delta x$, and occurring under the wave's influence, where $v$ is the average velocity of the propagating agent. We will focus on the cinematic properties of this mediating agent, to which should be associated a given subquantum wave. Using a unit normalizing constant, it will be for the propagating agent:

$$
E \approx v^{2}
$$

By differentiation we'll then have:

$$
\Delta E \approx 2 v \Delta v
$$

Since $\Delta E \approx 2 v \Delta v>v \Delta v$, expression (6') below, can be interpreted as a minimum generic energy interval for a causal agent, propagating between two interacting systems under the subquantum wave's influence. Thus we will consider for our purposes:

$$
\Delta E \approx v \Delta v
$$

Substituting (3) and (4) in (6), and still using the same unity normalizing constant for $\Delta p \approx \Delta v$, we get:

$$
\frac{1}{\Delta t} \sqrt{1-\Delta t^{2} / \sigma_{0 t}^{2}} \approx \frac{v}{\Delta x} \sqrt{1-\Delta x^{2} / \sigma_{0 x}^{2}}
$$

It is very important to note that in (7) we are not assuming on first terms that the linear relation $\Delta x \approx v \Delta t$ will hold. This is, of course, the relation normally attributable to the movement of a mediating agent between two interacting systems. We are, in fact, supposing that such a relation is but a particular case of a more general situation, where the spacetime properties of phenomena result from the subquantum wave properties. As such, in what follows, we will call $v$, as it is understood in the traditional linear relation $\Delta x \approx v \Delta t$, the "local velocity" of a mediating agent.

Again, it we will be assumed that spacetime reality results from a more fundamental one, the subquantum medium, and that the dependence of the former from the later can be explicitly formulated using a subquantum wave. In fact, using the left and right terms expressions in (7) and solving for $\Delta x$, one obtains the more general expression (8) relating $\Delta x$, the distance between two interacting systems, with the time interval $\Delta t$ during which the interaction occurs. This spacetime dependence, as it happens, depends primarily on the subquan- 
tum wave property domain, represented by $\sigma_{0 x}$, and on the subquantum property interconnectivity, represented by $\sigma_{0 t}$ :

$$
\Delta x \approx \frac{v \Delta t}{\sqrt{1-\frac{\Delta t^{2}}{\sigma_{0 t}^{2}}+\frac{v^{2} \Delta t^{2}}{\sigma_{0 x}^{2}}}}
$$

In (8) $\sigma_{0 x}$ represents the maximum extension of the mother wavelet, used in the wave packaging of the resulting overall subquantum wave, while $\sigma_{0 t}$ represents the longest measurable time of any interaction, associated to the same mother wavelet, and happening under the overall subquantum wave's influence. From expression (8) one can see that given two systems interacting with each other, through a mediating agent with local velocity $v$, the range of the interaction $\Delta x$ and its time interval $\Delta t$, in general will not depend strictly on $v$. Such relation will now also depend on the subquantum domain, represented by $\sigma_{0 x}$ in the subquantum wave, and on the subquantum interconnectivity, represented by $\sigma_{0 t}$ in the subquantum wave. That is,

$$
\Delta x \approx f\left(\Delta t, v, \sigma_{0 x}, \sigma_{0 t}\right)
$$

This is the reason we call (8) a generic spacetime relation. The form of such expression defines the spatial range of the interaction possibilities between two systems, for a given time interval. In other words, the overall subquantum wave has embedded in it the spacetime properties of whatever mediating signal is used to connect two interacting systems or even the kinematic properties of any given moving system, under the wave's influence. And in this way, one may infer that spacetime is indeed an emergent phenomenon from a prior subquantum medium.

\section{Explaining Entanglement and the Tunnel Effect}

Now, we will use the basic expression (8) to derive some general conclusions about how phenomena may be described to occur in space and time, for several cases of the subquantum domain $\sigma_{0 x}$ and of the subquantum interconnectivity $\sigma_{0 t}$. We start by noting that, by definition, for all $\Delta x$ :

$$
\Delta x \leq \sigma_{0 x}
$$

That is to say, the spatial extension of the mother wavelet is larger than the maximum spatial interval, for any interaction occurring under the wave's spatial influence.

Since by (8) one has in general $v \Delta t \leq \Delta x$, from (10), it results:

$$
v \Delta t \leq \sigma_{0 x}
$$

Again, by definition, it must be:

$$
\Delta t \leq \sigma_{0 t}
$$

That is to say, the temporal extension of the mother wavelet is larger than the maximum time interval, for any interaction occurring under the wave's spatial influence. 
Now, bearing in mind the relations (11) and (12), we will have the following situations.

1) Whenever both $\sigma_{0 x}$ and $\sigma_{0 t}$ become very large in (8), one regains the linear situation.

$$
\Delta x \approx v \Delta t
$$

2) Whenever the following condition holds:

$$
\frac{v^{2} \Delta t^{2}}{\sigma_{0 x}^{2}}-\frac{\Delta t^{2}}{\sigma_{0 t}^{2}}=0
$$

That is, whenever

$$
v=\frac{\sigma_{0 x}}{\sigma_{0 t}}
$$

Again, the linear case results and we have (13).

The linear case, of course, includes the relativistic saturated case, in which $v=c$, the velocity of light in the vacuum.

3) If we have a very large extended wave, that is, if $\sigma_{0 x} \rightarrow \infty$, but with $\sigma_{0 t} \approx \Delta t$, then we'll have:

$$
\Delta x \rightarrow \infty \forall \Delta t
$$

This may be interpreted as kind of an instantaneous action at distance, for very minute values of $\Delta t$ like in the gravitational Newtonian approach. As a special case in (15) above, we may even have $\sigma_{0 t}=\Delta t=0$. And thus the entanglement phenomenon between two correlated physical systems, apparently interacting very far apart in space, can be explained stating that they are under the influence of a common subquantum wave, for which the former special case of (15) holds in (8).

It should be recalled that in such a case the simple linear relation $\Delta x \approx v \Delta t$ will no longer hold and even looses any meaning. This makes clear the statement in 3.2 that the usual meaning for the velocity of causal agent will not apply in some situations. This situation, as already suggested, implies that spacetime behaviors are to be though as emergent phenomena from subquantum properties as, in fact, seems to be the case with entanglement.

4) Finally, we shall deal with the tunnel effect.

Elsewhere, and using the master nonlinear equation (2), it has been shown that the tunneling solutions inside the barrier are given by a wavelet expression [17]. Furthermore, by imposing continuity conditions for these solutions and their derivatives, we arrive at the expression that gives the resulting temporal separation between the incidence of the particle and its emergence at the other side of the barrier, the so-called transition time [17], by:

$$
T=\frac{\eta}{\hbar} \sqrt{\frac{E}{V-E}} \sigma_{0 x}^{2}
$$

With $E$ being the incident particle energy and $V$ the barrier height, $\eta$ a generic mass parameter associated with the particle and $\sigma_{0 x}$ the subquantum wave spatial component of its domain inside the barrier. That is, the maximum exten- 
sion of the wave that must match the barrier's length $L$, no matter how large. This formula is obviously only valid for the case where $V>E$, otherwise there will be no tunnel effect.

The most notable fact about this expression is that the transition time does not depend on the barrier length $L$. In other words, for the same wave and barrier's potential energy, one has:

$$
T=\text { constant }, \forall L
$$

Our aim is now to explain this surprising feature using (8). To do so we make the following identifications:

$$
T=\Delta t=\sigma_{0 t}, L=\Delta x=\sigma_{0 x}
$$

Which in turn, may be written by using (16) and (18):

$$
T=\Delta t=\sigma_{0 t}=\gamma \sigma_{0 x}^{2}
$$

With

$$
\gamma=\frac{\eta}{\hbar} \sqrt{\frac{E}{V-E}}
$$

By replacing (19) in (8), we get:

$$
\Delta x \approx \frac{v \gamma \sigma_{0 x}^{2}}{\sqrt{1-\frac{\left(\gamma \sigma_{0 x}^{2}\right)^{2}}{\left(\gamma \sigma_{0 x}^{2}\right)^{2}}+\frac{v^{2}\left(\gamma \sigma_{0 x}^{2}\right)^{2}}{\sigma_{0 x}^{2}}}}
$$

which, after simplification, yields:

$$
\Delta x \approx \sigma_{0 x}
$$

Using the second relation in (18), we have thus asserted that, in the tunnel effect, and for the same $\gamma$ :

$$
\text { If } \sigma_{0 t}=\gamma \sigma_{0 x}^{2} \text { and } \Delta t=\sigma_{0 t} \text { then } \Delta x \approx \sigma_{0 x} \text {, that is, } \Delta x \approx L
$$

from which expression (17) results.

In other words, the surprising independence between the barrier's length $L$ and the particle transition time $T$ in the tunnel effect results from relation (19) and from expression (8), the so called generic spacetime relation, valid for a subquantum wave.

\section{Conclusions}

In this work, we have assumed that spacetime may be understood as emergent phenomena, through subquantum waves, from the a priori basic physical reality identified with the subquantum medium. This however places a description problem, since human language and thought remain strongly rooted in space and time experiences. This difficulty was overcome by adopting what was called a minimum description procedure, where some primitive terms, still equated with space and time, were given a different interpretation and identified with subquantum properties. We have then used a set of generalized uncertainty rela- 
tions, derived in the context of wavelet analysis, to formulate a generic relation between space and time intervals under a subquantum wave domain of influence. Finally, we have used such relation to address entanglement phenomena and the tunnel effect.

The main conclusions we wish to convey with our efforts are the following:

1) In the most general sense, space and time, as a relation experienced by moving objects, do not have to be necessarily linear. Such relations will depend on the embedded properties of the subquantum waves which generate the spacetime arena where and while the phenomena under interest occur.

2) Thus space and time seem to be understandable as derived realities, from a prior subquantum realm. This means that the subquantum medium must not be equated with a pervading space itself, being a prior causal distinct reality.

3) From what has been asserted, the concept of superluminal velocity will not apply in the context of the entanglement and tunnel effect phenomena. A superluminal velocity would still imply a linear relationship between space and time.

4) Finally, one may also conclude that a given physical system may have, different spacetime properties in its different components, depending on how different subquantum waves are influencing it, that is, depending on the complexity and richness of the overall subquantum wave.

So we endow the overall subquantum wavefunction with the task of creating the spatial and chronological relations in which physical interactions occur, at all scales, both astronomical and microscopic. Depending on the arrangement of conditions in which some physical systems are observed, one may, in these special circumstances, reveal unusual phenomena that would be simplistically described, e.g., as superluminal, but that are in fact due to the invalidity of the averaging out of the spacetime relations one obtains in general.

\section{Acknowledgements}

This work has been funded by FCT (Portugal) through CFCUL (Project UID/FIL/ 00678/2013).

\section{Conflicts of Interest}

The authors declare no conflicts of interest regarding the publication of this paper.

\section{References}

[1] Bacciagaluppi, G. and Valentini, A. (2009) Quantum Theory at the Crossroads. Cambridge University Press, Cambridge, UK. https://doi.org/10.1017/CBO9781139194983

[2] Croca, J.R. (2003) Towards a Nonlinear Quantum Physics. World Scientific, London.

[3] Chui, C.K. (1992) An Introduction to Wavelets. Academic Press, New York.

[4] Kumar, P. (1997) Wavelet Analysis for Geophysical Applications. Review of Geophysics, 35, 385-412. https://doi.org/10.1029/97RG00427 
[5] Croca, J.R. (2007) Local Analysis by Wavelets versus Nonlocal Fourier Analysis. International Journal of Quantum Information, 5, 1-7. https://doi.org/10.1142/S0219749907002827

[6] Croca, J.R. (2013) Quantum Indeterminism: A Direct Consequence of Fourier Ontology. Proceedings Volume 8832, The Nature of Light: What are Photons? V, $88320 \mathrm{Y}$.

[7] Croca, J.R. (1995) On the Uncertainty Relations. In: van der Merwe, A. and Ferrero, M., Eds., Problems in Quantum Physics, Plenum.

[8] Croca, J.R. (1999) The Uncertainty Relations. Apeiron, 65, 151-165.

[9] Croca, J.R. and Selleri, F. (1999) Some Remarks Concerning Heisenberg's Indeterminacy Relations. Comm. Math. Theor. Physics, 2, 61-69.

[10] Bohr, N. (1985) Como Lectures, Collected Works. Vol. 6, North-Holand, Amsterdam.

[11] Castro, P., Croca, J.R., Gatta, M. and Moreira, R. (2017) Generalized Uncertainty Relations in Quantum Mechanics and the Principles of Completeness in Physics. Physical Science International Journal, 16, 1-9. https://doi.org/10.9734/PSIJ/2017/37038

[12] Croca, J.R. and Garuccio, A. (2010) Fourier Ontology versus Wavelet Local Analysis a Test for the General Validity of Orthodox Mechanics against the Nonlinear Causal Quantum Physics. Advanced Science Letters, 3, 214-217. https://doi.org/10.1166/asl.2010.1114

[13] de Broglie, L. (1969) The Current Interpretation of Wave Mechanics: A Critical Study. Elsevier, Amsterdam.

[14] Croca, J.R., Castro, P., Gatta, M., Cardoso, A. and Moreira, R. (2017) Pilot-Wave Gravity and the Titius-Bode Law. New Horizons in Mathematical Physics, 75-81.

[15] Castro, P., Croca, J.R., Moreira, R. and Gatta, M. (2017) On the Foundations of Eurhythmic Physics: A Brief Non Technical Survey. International Journal of Philosophy, 5, 50-53.

[16] Castro, P., Croca, J.R. and Moreira, R. (2018) An Introduction to Eurhythmic Philosophy. Brolly. Journal of Social Sciences, 1, 19-31.

[17] Croca, J.R. (2015) Eurhythmic Physics, or Hyperphysics, the Unification of Physics. Lambert Academic Publishing, Berlin. 